\title{
Assessment of microsatellites in estimating inter- and intraspecific variation among Neotropical Crocodylus species
}

\author{
A. Bashyal ${ }^{1 *}$, B.A. Gross ${ }^{1 *}$, M. Venegas-Anaya ${ }^{1,2}$, F. Lowrance $^{1}$ and \\ L.D. Densmore III ${ }^{1}$ \\ ${ }^{1}$ Department of Biological Sciences, Texas Tech University, Lubbock, \\ TX, USA \\ ${ }^{2}$ Smithsonian Tropical Research Institute, Balboa, Ancón, \\ Republic of Panama \\ *These authors contributed equally to this study. \\ Corresponding author: M. Venegas-Anaya \\ E-mail: dracocodrilo@hotmail.com
}

Genet. Mol. Res. 13 (3): 5492-5502 (2014)

Received June 17, 2013

Accepted February 3, 2014

Published July 25, 2014

DOI http://dx.doi.org/10.4238/2014.July.25.2

\begin{abstract}
We tested microsatellites that were developed for the saltwater crocodile (Crocodylus porosus) for cross-species amplification and to provide an estimate of inter- and intraspecific variation among four species of Neotropical crocodiles (C. rhombifer, $C$. intermedius, $C$. acutus, and $C$. moreletii). Our results indicated that with the exception of 2 loci in $C$. intermedius, all 10 microsatellite loci were successfully amplified in the 4 species, producing a set of variably sized alleles that ranged in number between 2 and 14 alleles per locus. Similarly, private alleles (i.e., unique alleles) also were reported in all 4 species for at least 3 loci. The mean observed and expected heterozygosities (averaged across species for all 10 loci combined) ranged from 0.39 to 0.77 and from 0.44 to 0.78 , respectively. In addition to this, we evaluated these microsatellites in 2 populations of $C$. acutus and $C$. moreletii to assess their utility in estimating intraspecific levels of polymorphisms. These
\end{abstract}


microsatellites also showed considerable allelic variation in population level analysis. The set of 10 microsatellite loci in our study had the potential to be used as a tool in population and conservation genetic studies of Neotropical crocodiles.

Key words: Microsatellites; Crocodiles; Cross-species amplification; Neotropical crocodiles

\section{INTRODUCTION}

Microsatellites are repetitive sequences of 1 to $6 \mathrm{bp}$ of DNA that are mostly found in the non-coding regions of the eukaryotic genome and are known to have high mutation rates that lead to greater allelic variability and high levels of polymorphism (Wright and Bentzen, 1994). Because they are hypervariable and occur ubiquitously, microsatellites have widely been used as tools for a variety of fields of study such as population and conservation genetics, molecular ecology (mating behavior and gene flow), and wildlife DNA forensic analyses (Glenn et al., 1998; Fitzsimmons et al., 2001; Davis et al., 2001, 2002; Dever et al., 2002; Avise, 2004; Anmarkrud et al., 2008).

Despite their widespread application in this discipline, one drawback of using microsatellites as genetic markers has been the development of primers, which can involve both considerable time and resources. Fortunately, a number of microsatellite loci are conserved enough across species to be useful in interspecific and intraspecific marker comparisons (Glenn et al., 1998). Successful cross-species amplification of microsatellites has been reported from groups such as mammals (Williamson et al., 2002; Gunn et al., 2005), birds (Primmer et al., 1996, 2005), insects (Wilson et al., 2004), fish (Scribner et al., 1996; King et al., 2001), snakes (King, 2009; Vandewege et al., 2012), and crocodilians (Dever and Densmore III, 2001; Zucoloto et al., 2006; Weaver et al., 2008; Milián-Garcia et al., 2011; Rodriguez et al., 2008, 2011).

The "Neotropical crocodiles" consist of 4 species: the American crocodile (Crocodylus acutus), Cuban crocodile (C. rhombifer), Orinoco crocodile (C. intermedius), and Morelet's crocodile ( $C$. moreletii). Of the 4 species, 2 (C. rhombifer and C. intermedius) are considered critically endangered (Crocodile Specialist Group, 1996; Targarona et al., 1996), whereas C. acutus is considered vulnerable (Ponce-Campos et al., 2012) and C. moreletii is regarded as lower concern (Cedeño-Vázquez et al., 2012). Given their conservation status, having a larger number of successfully amplified microsatellite loci that exhibit high levels of polymorphism at the inter- and intraspecific levels in these Neotropical crocodiles could serve as an important conservation tool.

Recently, Miles et al. (2009a) developed 253 novel microsatellite loci from the saltwater crocodile (C. porosus) library and tested 82 microsatellites for cross-species amplification in 18 species of crocodilians (Miles et al., 2009b). The set of microsatellites included in our study consisted of 5 loci that were successfully cross-species amplified by Miles et al. (2009b) and 5 loci tested herein for the first time for cross-species amplification in Neotropical crocodiles from Miles et al. (2009a).

We evaluated these 10 microsatellite loci for cross-species amplification in all 4 species of Neotropical crocodiles and in populations from 2 of these species, C. acutus (from 
Panama and Mexico) and C. moreletii (from Mexico and Belize). The objectives of our study were to: a) test microsatellite primers that were developed for $C$. porosus for cross-species amplification in Neotropical crocodiles, b) assess the utility of these microsatellites in estimating levels of inter- and intraspecific polymorphism among the Neotropical crocodiles, and c) identify private alleles (alleles that are unique to a particular species or population) at the species and population levels that can be used to identify species and populations.

\section{MATERIAL AND METHODS}

Tissue samples were taken from 5 C. rhombifer, 5 C. intermedius, 26 C. acutus (16 from Panama and 10 from Mexico), and 45 C. moreletii (32 from Belize and 13 from Mexico) individuals. Total genomic DNA was isolated by proteinase $\mathrm{K}$ digestion and extracted using the cetyltrimethylammonium bromide-phenol-chloroform technique (Sambrook et al., 1989; Palumbi, 1996). We first chose 40 microsatellite loci from Miles et al. (2009a) that were reported to have high levels of polymorphism. We then tested those 40 loci for cross-species amplification in C. moreletii and C. acutus, and only 10 loci showed consistent amplification. The 10 microsatellite loci that showed cross-species amplification in C. moreletii and C. acutus were further tested for cross-species amplification in other Neotropical crocodiles using a 2-primer polymerase chain reaction (PCR). Sequence-specific forward primers from Miles et al. (2009a) were modified by adding a 5'-M13 tail, whereas the reverse primers were used as they were designed. The M13 tail is a complementary oligonucleotide sequence of a universal M13 fluorescence nucleic acid polymer (5'-CACGACGTTGTAAAACGAC-3'). The final amplification was performed using "3-primer competition" PCR (Schuelke, 2000) (Tables 1 and 2). Fluorescent dye labels (FAM, VIC, NED, and PET; Applied Biosystems, USA) were used to label the universal M13 nucleotide 5'-tail (Table 1). The PCR products were electrophoresed on $2 \%$ agarose gels and run for $20 \mathrm{~min}$ at $70-75 \mathrm{~V}$.

PCRs were carried out using 2 different programs (Table 1). The TD-65 program (modified from Miles et al., 2009a) consisted of an initial denaturation step of $95^{\circ} \mathrm{C}$ for $3 \mathrm{~min}$; 4 cycles of $95^{\circ} \mathrm{C}$ for $30 \mathrm{~s}, 65^{\circ} \mathrm{C}$ for $30 \mathrm{~s}$, and $72^{\circ} \mathrm{C}$ for $45 \mathrm{~s} ; 4$ cycles of $95^{\circ} \mathrm{C}$ for $30 \mathrm{~s}, 62^{\circ} \mathrm{C}$ for $30 \mathrm{~s}$, and $72^{\circ} \mathrm{C}$ for $45 \mathrm{~s} ; 8$ cycles of $95^{\circ} \mathrm{C}$ for $30 \mathrm{~s}, 60^{\circ} \mathrm{C}$ for $30 \mathrm{~s}$, and $72^{\circ} \mathrm{C}$ for $45 \mathrm{~s} ; 24$ cycles of $95^{\circ} \mathrm{C}$ for $30 \mathrm{~s}, 55^{\circ} \mathrm{C}$ for $30 \mathrm{~s}$, and $72^{\circ} \mathrm{C}$ for $45 \mathrm{~s}$; and a final extension step at $72^{\circ} \mathrm{C}$ for $5 \mathrm{~min}$. Similarly, the T-55 program consisted of an initial denaturation step of $94^{\circ} \mathrm{C}$ for $3 \mathrm{~min} ; 34$ cycles of $94^{\circ} \mathrm{C}$ for $40 \mathrm{~s}, 55^{\circ} \mathrm{C}$ for $40 \mathrm{~s}$, and $72^{\circ} \mathrm{C}$ for $60 \mathrm{~s}$; and a final extension step at $72^{\circ} \mathrm{C}$ for $5 \mathrm{~min}$. An ABI 3100 Avant genetic analyzer (Applied Biosystems) was used to detect and size fragments that were fluorescently labeled with the reaction (for each microsatellite), which consisted of a final volume of $12 \mu \mathrm{L}$ including $0.6 \mu \mathrm{L} \mathrm{LIZ} \mathrm{(500)} \mathrm{standard,} 1.5 \mu \mathrm{L}$ PCR product, and $9.9 \mu \mathrm{L}$ formamide.

Electrophoretograms were analyzed in GENEMAPPER 3.7 (Applied Biosystems) using the following analysis method: the size calling parameter was set at local Southern, the signal levels for homozygous and heterozygous minimum peak height were set at 140 and 85 , respectively (following the manufacturer guideline for ABI 3100), and the minimum peak height ratio was set at 0.2 . These parameters were selected to identify any stutter peaks that might be present and also to identify the alleles that might have unequal amplification and thus were not scored. During the amplification process of microsatellites, the formation of stutter peaks is usually caused by the effects of polymerase slippage, which can ultimately cause a single or multiple repeat units to be skipped or added to the strands that are produced during 
Table 1. Primer sequences (provided by Miles et al., 2009a), fluorescent dye labels, and PCR conditions for the 10 microsatellite loci.

\begin{tabular}{|c|c|c|c|}
\hline Locus & Primers (5' to $\left.3^{\prime}\right)$ & Fluorescent dye label & PCR conditions \\
\hline CpDi06 & $\begin{array}{l}\text { F: TGTTGGGCACTTTGAAC } \\
\text { R: GTTTAAGAAAAATGGTGGAAAAC }\end{array}$ & PET & $\mathrm{T}-55$ \\
\hline $\mathrm{CpP} 2201$ & $\begin{array}{l}\text { F: CAAGTGACCCCTTTTCAG } \\
\text { R: GTTTATTTCTTGTTTGGCACATC }\end{array}$ & VIC & $\mathrm{T}-55$ \\
\hline СpP2202* & $\begin{array}{l}\text { F: GCAACAAAAGACCTTGACA } \\
\text { R: GTTTGGTTGGGTGGAATTATATAC }\end{array}$ & NED & $\mathrm{T}-55$ \\
\hline СpP208* & $\begin{array}{l}\text { F: CACATGGCTTTTGTTCTGAG } \\
\text { R: GTTTCCTGCAAAATGTTCTCCTA }\end{array}$ & FAM & TD-65 \\
\hline CpP1306* & $\begin{array}{l}\text { F: TTCTCTCTAGGAGCCACTCAC } \\
\text { R: GTTTAGGGAGACATCTAGGAAGAAC }\end{array}$ & NED & TD-65 \\
\hline CpP801 & $\begin{array}{l}\text { F: TTGGCATTAGATTGGTAGAC } \\
\text { R: GTTTCTATGCCAAAGCTACAAC }\end{array}$ & VIC & T-55 \\
\hline CpDi04* & $\begin{array}{l}\text { F: TTCTAAACAGTCCAGGATGA } \\
\text { R: GTTTAAATTTCACTAGATGCCATAA }\end{array}$ & FAM & $\mathrm{T}-55$ \\
\hline CpP815 & $\begin{array}{l}\text { F: GGTTAAGTGCCACACAAGT } \\
\text { R: GTTTGGCCAATTTCTAATGAA }\end{array}$ & NED & $\mathrm{T}-55$ \\
\hline CpP314* & $\begin{array}{l}\text { F: GAAATGCCACTAATACACACA } \\
\text { R: GTTCCAATTCTTCAGGTCCTTAT }\end{array}$ & FAM & $\mathrm{T}-55$ \\
\hline CpP4311 & $\begin{array}{l}\text { F: GGCTGCTCTGTGTTTG } \\
\text { R: GTTTGGGTTTAGCATCATGT }\end{array}$ & VIC & TD-65 \\
\hline CpDi06 & $\begin{array}{l}\text { F: TGTTGGGCACTTTGAAC } \\
\text { R: GTTTAAGAAAAATGGTGGAAAAC }\end{array}$ & PET & T-55 \\
\hline $\mathrm{CpP} 2201$ & $\begin{array}{l}\text { F: CAAGTGACCCCTTTTCAG } \\
\text { R: GTTTATTTCTTGTTTGGCACATC }\end{array}$ & VIC & $\mathrm{T}-55$ \\
\hline $\mathrm{CpP} 2202^{*}$ & $\begin{array}{l}\text { F: GCAACAAAAGACCTTGACA } \\
\text { R: GTTTGGTTGGGTGGAATTATATAC }\end{array}$ & NED & $\mathrm{T}-55$ \\
\hline CpP208* & $\begin{array}{l}\text { F: CACATGGCTTTTGTTCTGAG } \\
\text { R: GTTTCCTGCAAAATGTTCTCCTA }\end{array}$ & FAM & TD-65 \\
\hline CpP1306* & $\begin{array}{l}\text { F: TTCTCTCTAGGAGCCACTCAC } \\
\text { R: GTTTAGGGAGACATCTAGGAAGAAC }\end{array}$ & NED & TD-65 \\
\hline CpP801 & $\begin{array}{l}\text { F: TTGGCATTAGATTGGTAGAC } \\
\text { R: GTTTCTATGCCAAAGCTACAAC }\end{array}$ & VIC & T-55 \\
\hline CpDi04* & $\begin{array}{l}\text { F: TTCTAAACAGTCCAGGATGA } \\
\text { R: GTTTAAATTTCACTAGATGCCATAA }\end{array}$ & FAM & T-55 \\
\hline CpP815 & $\begin{array}{l}\text { F: GGTTAAGTGCCACACAAGT } \\
\text { R: GTTTGGCCAATTTCTAATGAA }\end{array}$ & NED & T-55 \\
\hline СpP314* & $\begin{array}{l}\text { F: GAAATGCCACTAATACACACA } \\
\text { R: GTTCCAATTCTTCAGGTCCTTAT }\end{array}$ & FAM & T-55 \\
\hline CpP4311 & $\begin{array}{l}\text { F: GGCTGCTCTGTGTTTG } \\
\text { R: GTTTGGGTTTAGCATCATGT }\end{array}$ & VIC & TD-65 \\
\hline
\end{tabular}

*Previously untested microsatellites from Miles et al. (2009a).

Table 2. Reaction mix for amplification of the 10 microsatellite loci.

\begin{tabular}{lcc}
\hline Reagents & \multicolumn{2}{c}{ Final concentration of each reagent for the respective loci } \\
\cline { 2 - 3 } & CpDi06, CpP2201, & CpP1306, CpP801, CpDi04, \\
& CpP2202, CpP208 & $15, \mathrm{CpP} 314, \mathrm{CpP} 4311$ \\
\hline Taq buffer & $1 \mathrm{X}$ & $2 \mathrm{mM}$ \\
$\mathrm{MgCl}_{2}$ & $2 \mathrm{mM}$ & $0.52 \mathrm{mM}$ each \\
dNTPs & $0.52 \mathrm{mM}$ each & $0.2 \mu \mathrm{M}$ \\
Forward primer & $0.2 \mu \mathrm{M}$ & $0.2 \mu \mathrm{M}$ \\
Reverse primer & $0.2 \mu \mathrm{M}$ & $0.1 \mu \mathrm{M}$ \\
M13 & $0.1 \mu \mathrm{M}$ & $0.028 \mathrm{U} / \mu \mathrm{L}$ \\
Taq Polymerase* & $0.03 \mathrm{U} / \mu \mathrm{L}$ & $0.8 \mathrm{ng} / \mu \mathrm{L}$ \\
Template DNA & $1 \mathrm{ng} / \mu \mathrm{L}$ & \\
\hline
\end{tabular}

*Qiagen Taq polymerase. 
PCR (Watzinger et al., 2006). Using the fluorescent-based PCR technique, stutter peaks can be recognized as additional products of the PCR that have a lower peak height; the majority of the time, they tend to appear 1 repeat unit downstream from the true allele (Watzinger et al., 2006).

CERVUS 3.0 (Marshall et al., 1998) was used to estimate the number of alleles $(A)$, observed heterozygosity $\left(H_{\mathrm{O}}\right)$, and expected heterozygosity $\left(H_{\mathrm{E}}\right)$. GENALEX 6.0 (Peakall and Smouse, 2006) was used to estimate the allele frequency, number of private alleles $\left(A_{\mathrm{p}}\right)$, and probability of identity $(P I)$ by locus.

\section{RESULTS}

Cross-species amplification was observed among all possible PCRs involving C. acutus, C. moreletii, and C. rhombifer, and it was observed at the $80 \%$ level for $C$. intermedius. With only 2 exceptions (CpDi06 and CpP801 for C. intermedius), all 10 microsatellite loci were successfully amplified in all 4 species of Neotropical crocodiles. All amplified loci produced a variety of alleles and appeared to be polymorphic in all 4 species with the exception of 1 locus (CpP2202) in C. rhombifer, which was found to be monomorphic.

Stutter peaks of all 10 loci were present in low frequencies for one or more species (Table 3). The presence of stutter peaks can potentially create incorrect scoring between alleles, where size differences are small, and can lead to calling true-heterozygotes as pseudohomozygotes or the opposite (Skotheim et al., 2001). Using an appropriate analysis method in GENEMAPPER 3.7 (see above in Material and Methods) to analyze the electrophoretograms, we were able to identify stutter peaks and any other alleles that were not scored due to unequal amplification, which reduced the misidentification of alleles.

A total of 94 different alleles were found among the 10 loci combined for all species. The $A$ value ranged from 2 to 14 with an overall mean of 4.95 (Table 3). The estimates of $H_{O}$ and $H_{E}$ ranged from 0.39 to 0.77 and 0.44 to 0.78 , respectively, with a mean of 0.62 and 0.61 , respectively (Table 3 ). The $P I$ by locus (the probability that 2 individuals drawn at random from a population will have the same genotype at multiple loci) ranged from 0.08 to 0.38 with a mean of 0.18 (Table 3). Diagnostic private (unique) alleles were found in all 4 species for at least 3 loci (Table 3). One interesting observation was that, although only 5 individuals of $C$. rhombifer were genotyped, there were a total of 33 alleles and 8 private alleles reported for this species, further demonstrating the hyper-variability of these microsatellites.

A finer scale population-level analyses (Tables 4 and 5) showed that all the microsatellite loci were polymorphic and that there was considerable allelic variation between the 2 populations of $C$. acutus from Panama (mean 4.4 alleles per locus) and Mexico (mean 4.8 alleles per locus), as well as between the 2 populations of $C$. moreletii from Mexico (mean of 4 alleles per locus) and Belize (mean 6.6 alleles per locus). Private alleles were reported in each population for at least 3 loci (Table 4). Although the frequencies of the private alleles were generally low, 2 alleles (allele 242 of locus CpDi06 and allele 149 of locus CpP801) in the $C$. acutus population from Panama had reasonably high frequencies ( 0.47 for both; Table 5$)$. The mean $H_{\mathrm{O}}$ was higher in the population of C. acutus from Mexico (0.70) than that from Panama (0.61; Table 4); however, there was not a statistically significant difference between the mean $H_{\mathrm{O}}$ of these 2 populations $(\mathrm{P}>0.05)$. Similarly, the mean $H_{O}$ was higher in the population of C. moreletii from Belize (0.76) than that from Mexico (0.70; Table 4); however, there was not a statistically significant difference between the mean $H_{O}$ of these 2 populations $(\mathrm{P}>0.05)$. 
Table 3. Number of alleles per locus $(A)$, number of private alleles per locus $\left(A_{\mathrm{p}}\right)$, probability of identity $(P I)$, size range of the PCR products, observed heterozygosity $\left(H_{\mathrm{O}}\right)$, and expected heterozygosity $\left(H_{\mathrm{E}}\right)$ of 10 microsatellite loci in each of the 4 species of Neotropical crocodiles.

\begin{tabular}{|c|c|c|c|c|c|c|c|}
\hline Locus & Species & $A$ & $A_{\mathrm{p}}$ & $P I$ & Size (bp) & $H_{\mathrm{O}}$ & $H_{\mathrm{E}}$ \\
\hline \multirow[t]{7}{*}{$\overline{\mathrm{CpP} 2201}$} & C. rhombifer* & 3 & 1 & 0.08 & $183-235$ & 0.2 & 0.64 \\
\hline & C. intermedius & 4 & 1 & 0.26 & $179-227$ & 0.4 & 0.53 \\
\hline & C. acutus & 6 & 1 & 0.08 & $191-235$ & 1 & 0.73 \\
\hline & C. moreletii & 4 & 0 & 0.18 & $191-227$ & 0.86 & 0.57 \\
\hline & Mean & 4.25 & - & 0.15 & - & 0.62 & 0.62 \\
\hline & & $A$ & $A_{\mathrm{p}}$ & $P I$ & Size (bp) & $H_{\mathrm{O}}$ & $H_{\mathrm{E}}$ \\
\hline & C. rhombifer & 1 & 0 & 0.39 & 184 & - & - \\
\hline \multirow[t]{6}{*}{ CpP2202 } & C. intermedius* & 3 & 1 & 0.38 & $180-196$ & 0.6 & 0.69 \\
\hline & C. acutus & 2 & 0 & 0.18 & $188-200$ & 0.96 & 0.51 \\
\hline & C. moreletii & 3 & 0 & 0.18 & $188-200$ & 0.96 & 0.58 \\
\hline & Mean & 2.25 & - & 0.28 & - & 0.63 & 0.45 \\
\hline & & $A$ & $A_{\mathrm{p}}$ & $P I$ & Size (bp) & $H_{0}$ & $H_{\mathrm{F}}$ \\
\hline & C. rhombifer* & 3 & $2^{p}$ & 0.05 & $218-236$ & 0.6 & 0.51 \\
\hline \multirow[t]{6}{*}{ CpDi06 } & C. intermedius & - & - & - & - & - & - \\
\hline & C. acutus & 10 & 7 & 0.18 & $224-264$ & 0.92 & 0.76 \\
\hline & C. moreletii & 5 & 1 & 0.18 & $226-248$ & 0.77 & 0.55 \\
\hline & Mean & 4.5 & - & 0.10 & - & 0.57 & 0.46 \\
\hline & & $A$ & $A_{\mathrm{p}}$ & $P I$ & Size (bp) & $H_{0}$ & $H_{\mathrm{F}}$ \\
\hline & C. rhombifer* & 3 & 1 & 0.39 & $179-191$ & 0.2 & 0.51 \\
\hline \multirow[t]{6}{*}{ CpP208 } & C. intermedius & 2 & 0 & 0.38 & $179-191$ & 0.8 & 0.53 \\
\hline & C. acutus* & 2 & 0 & 0.38 & 179-191 & 0.92 & 0.51 \\
\hline & C. moreletii & 2 & 0 & 0.38 & $179-191$ & 0.71 & 0.46 \\
\hline & Mean & 2.25 & - & 0.38 & - & 0.66 & 0.50 \\
\hline & & $A$ & $A_{\mathrm{p}}$ & $P I$ & Size (bp) & $H_{0}$ & $H_{\mathrm{F}}$ \\
\hline & C. rhombifer & 5 & $2^{p}$ & 0.25 & $234-270$ & 0.6 & 0.82 \\
\hline \multirow[t]{6}{*}{ СpP314 } & C. intermedius & 4 & 0 & 0.13 & $246-266$ & 1 & 0.78 \\
\hline & C. acutus & 8 & 0 & 0.09 & $246-282$ & 0.5 & 0.80 \\
\hline & C. moreletii & 10 & 4 & 0.09 & $238-286$ & 0.64 & 0.73 \\
\hline & Mean & 6.75 & - & 0.14 & - & 0.69 & 0.78 \\
\hline & & $A$ & $A_{\mathrm{p}}$ & $P I$ & Size (bp) & $H_{\mathrm{O}}$ & $H_{\mathrm{E}}$ \\
\hline & C. rhombifer & 4 & $\begin{array}{c}\mathrm{P}_{\mathrm{p}} \\
0\end{array}$ & 0.39 & $195-211$ & 1 & 0.73 \\
\hline \multirow{6}{*}{ CpP4311 } & C. intermedius* & 2 & 0 & 0.18 & $187-199$ & 1 & 0.56 \\
\hline & C. acutus & 4 & 0 & 0.14 & $195-219$ & 0.28 & 0.54 \\
\hline & C. moreletii & 7 & 2 & 0.14 & $175-219$ & 0.58 & 0.59 \\
\hline & Mean & 4.25 & - & 0.21 & - & 0.72 & 0.61 \\
\hline & & $A$ & $A_{\mathrm{p}}$ & $P I$ & Size (bp) & $H_{0}$ & $H_{\mathrm{F}}$ \\
\hline & C. rhombifer & 4 & 0 & 0.11 & $218-242$ & 0.8 & 0.80 \\
\hline \multirow[t]{6}{*}{ CpP815 } & C. intermedius & 2 & 0 & 0.08 & $222-242$ & 1 & 0.56 \\
\hline & C. acutus & 10 & 4 & 0.05 & $206-246$ & 0.46 & 0.85 \\
\hline & C. moreletii & 6 & 0 & 0.09 & $218-246$ & 0.82 & 0.71 \\
\hline & Mean & 5.5 & - & 0.08 & - & 0.77 & 0.73 \\
\hline & & A & $A_{\mathrm{p}}$ & $P I$ & Size (bp) & $H_{\mathrm{O}}$ & $H_{\mathrm{E}}$ \\
\hline & C. rhombifer & 2 & 0 & 0.22 & $157-169$ & $\begin{array}{c}x_{0} \\
0.4\end{array}$ & $\begin{array}{l}0.36 \\
0.5\end{array}$ \\
\hline \multirow[t]{6}{*}{ CpP801 } & C. intermedius & - & - & - & - & - & - \\
\hline & C. acutus & 10 & 2 & 0.05 & $145-185$ & 0.56 & 0.85 \\
\hline & C. moreletii & 14 & 6 & 0.03 & $141-201$ & 0.75 & 0.86 \\
\hline & Mean & 6.5 & - & 0.08 & - & 0.43 & 0.52 \\
\hline & & $A$ & $A_{\mathrm{p}}$ & $P I$ & Size (bp) & $H_{0}$ & $H_{\mathrm{E}}$ \\
\hline & C. rhombifer* & 5 & $2^{\mathrm{P}}$ & 0.23 & $156-170$ & $\begin{array}{c}0.6 \\
0.6\end{array}$ & $\begin{array}{l}1 \\
0.87\end{array}$ \\
\hline \multirow{5}{*}{ CpDi04 } & C. intermedius* & 3 & 2 & 0.46 & $164-176$ & 0.2 & 0.73 \\
\hline & C. acutus & 6 & 1 & 0.18 & $142-166$ & 0.17 & 0.49 \\
\hline & C. moreletii & 8 & 1 & 0.14 & $142-166$ & 0.59 & 0.64 \\
\hline & Mean & 5.5 & - & 0.25 & - & 0.39 & 0.68 \\
\hline & & $A$ & $A_{\mathrm{p}}$ & $P I$ & Size (bp) & $H_{0}$ & $H_{\mathrm{F}}$ \\
\hline \multirow{5}{*}{ CpP1306 } & C. rhombifer & 3 & $\begin{array}{c}\mathrm{p}_{\mathrm{p}} \\
0\end{array}$ & 0.16 & 93-109 & $\begin{array}{c}0 \\
0.4\end{array}$ & $\begin{array}{l}\mathrm{F}_{\mathrm{E}} \\
0.60\end{array}$ \\
\hline & C. intermedius & 6 & 0 & 0.11 & $85-121$ & 1 & 0.84 \\
\hline & C. acutus & 9 & 1 & 0.05 & $81-149$ & 0.63 & 0.78 \\
\hline & C. moreletii & 13 & 4 & 0.05 & $77-153$ & 0.81 & 0.83 \\
\hline & Mean & 7.75 & - & 0.09 & - & 0.71 & 0.76 \\
\hline
\end{tabular}

*Absence of stutter peaks in that species for that particular locus. 
Table 4. Number of alleles per locus $(A)$, number of private alleles per locus $\left(A_{\mathrm{p}}\right)$, probability of identity $(P I)$, observed heterozygosity $\left(H_{\mathrm{O}}\right)$, and expected heterozygosity $\left(H_{\mathrm{E}}\right)$ of 10 microsatellite loci in each of the 2 populations of Crocodylus acutus and C. moreletii.

\begin{tabular}{|c|c|c|c|c|c|c|c|c|c|c|c|c|c|c|c|c|c|c|c|c|}
\hline \multirow[b]{2}{*}{ Locus } & \multicolumn{5}{|c|}{ C. acutus - Panama $(\mathrm{N}=16)$} & \multicolumn{5}{|c|}{ C. acutus - Mexico $(\mathrm{N}=10)$} & \multicolumn{5}{|c|}{ C. moreletii - Mexico $(\mathrm{N}=13)$} & \multicolumn{5}{|c|}{ C. moreletii - Belize $(\mathrm{N}=32)$} \\
\hline & $A$ & $A_{\mathrm{p}}$ & $P I$ & $H_{\mathrm{o}}$ & $H_{\mathrm{E}}$ & $A$ & $A_{\mathrm{P}}$ & $P I$ & $H_{\mathrm{O}}$ & $H_{\mathrm{E}}$ & $A$ & $A_{\mathrm{P}}$ & $P I$ & $H_{\mathrm{o}}$ & $H_{\mathrm{E}}$ & $A$ & $A_{\mathrm{P}}$ & $P I$ & $H_{\mathrm{o}}$ & $H_{\mathrm{E}}$ \\
\hline СpP2201 & 3 & 1 & 0.30 & 1.00 & 0.57 & 5 & 0 & 0.11 & 1.00 & 0.79 & 3 & 0 & 0.28 & 1.00 & 0.59 & 3 & 0 & 0.30 & 0.81 & 0.56 \\
\hline CpP2202 & 2 & 0 & 0.38 & 1.00 & 0.52 & 2 & 0 & 0.38 & 0.90 & 0.52 & 3 & 0 & 0.32 & 1.00 & 0.56 & 3 & 0 & 0.27 & 0.94 & 0.59 \\
\hline CpDi06 & 2 & 1 & 0.38 & 0.94 & 0.51 & 9 & 5 & 0.04 & 0.89 & 0.90 & 2 & 0 & 0.38 & 0.75 & 0.52 & 5 & 2 & 0.27 & 0.78 & 0.57 \\
\hline CpP208 & 2 & 0 & 0.38 & 0.94 & 0.51 & 2 & 0 & 0.38 & 0.89 & 0.52 & 2 & 0 & 0.38 & 0.92 & 0.52 & 2 & 0 & 0.42 & 0.63 & 0.44 \\
\hline CpP314 & 6 & 1 & 0.22 & 0.44 & 0.59 & 5 & 0 & 0.14 & 0.60 & 0.72 & 4 & 0 & 0.16 & 0.54 & 0.71 & 10 & 4 & 0.11 & 0.69 & 0.72 \\
\hline СpP4311 & 4 & 0 & 0.42 & 0.19 & 0.38 & 2 & 0 & 0.49 & 0.44 & 0.37 & 4 & 0 & 0.46 & 0.39 & 0.35 & 7 & 3 & 0.16 & 0.66 & 0.66 \\
\hline CpP815 & 8 & 3 & 0.09 & 0.31 & 0.78 & 6 & 1 & 0.08 & 0.70 & 0.81 & 4 & 1 & 0.16 & 0.77 & 0.72 & 5 & 0 & 0.15 & 0.84 & 0.70 \\
\hline CpP801 & 8 & 2 & 0.09 & 0.50 & 0.76 & 6 & 0 & 0.14 & 0.67 & 0.72 & 8 & 2 & 0.06 & 0.58 & 0.85 & 11 & 3 & 0.04 & 0.81 & 0.86 \\
\hline CpDi04 & 2 & 0 & 0.88 & 0.06 & 0.06 & 6 & 1 & 0.16 & 0.43 & 0.68 & 4 & 0 & 0.18 & 0.30 & 0.69 & 8 & 3 & 0.21 & 0.68 & 0.63 \\
\hline CpP1306 & 7 & 1 & 0.12 & 0.69 & 0.72 & 5 & 0 & 0.12 & 0.50 & 0.77 & 6 & 1 & 0.11 & 0.80 & 0.78 & 12 & 3 & 0.06 & 0.81 & 0.83 \\
\hline Mean & 4.4 & - & 0.33 & 0.61 & 0.54 & 4.8 & - & 0.20 & 0.70 & 0.68 & 4.0 & - & 0.25 & 0.71 & 0.63 & 6.6 & - & 0.20 & 0.77 & 0.66 \\
\hline
\end{tabular}

\section{DISCUSSION}

All 10 microsatellites that were tested in our study were informative and produced a variety of both shared and private alleles at both the cross-species and inter-population levels. The number of alleles in a population (allelic richness) and the number of unique alleles in a population (private allelic richness) are considered to be useful for many conservation and population genetics applications (Kalinowski, 2004) and can be used to infer the evolutionary history of a population (Castric and Bernatchez, 2003). Together, these measures of genetic diversity can be beneficial in identifying populations of Neotropical crocodiles that exhibit low levels of genetic diversity so that specific conservation actions can be undertaken for those populations. Private allelic richness will also be informative in distinguishing between species and between different populations within a species. Despite its utility, there is no doubt that private allelic richness is a function of sample size (Kalinowski, 2004). Thus, additional sampling may reveal that many of the alleles that are currently recognized as private are actually not private.

The microsatellite loci that were used in our study, combined with previously developed microsatellite libraries (Fitzsimmons et al., 2001; Miles et al., 2009a,b), can be used to help conserve and manage these 4 species of Neotropical crocodiles. Microsatellites that can be successfully amplified in cross-species reactions can aid managers in identification of purebred populations as well as hybrid and admixed individuals. Because illegal hunting for skin and meat is a major threat to all Neotropical crocodiles, these markers can also be used as forensic tools for law enforcement to facilitate stricter regulations against illegal hunting (Targarona et al., 1996; Cedeño-Vázquez et al., 2012; Ponce-Campos et al., 2012). This suite of microsatellites will also help reduce the time and resources needed to characterize the genetic fingerprint of any particular species, thus adding microsatellites to the list of conservation tools that can be used in the management of Neotropical and other crocodile species. 
Table 5. Allele frequencies of 10 microsatellite loci in populations of Crocodylus acutus and C. moreletii.

\begin{tabular}{|c|c|c|c|c|c|}
\hline Locus & Allele & Pop. $1^{\mathrm{a}}$ & Pop. $2^{\text {b }}$ & Pop. $3^{c}$ & Pop. 4 \\
\hline \multicolumn{6}{|c|}{$\overline{\mathrm{CpP} 2201}$} \\
\hline & 191 & 0.06 & - & - & 0.06 \\
\hline & 211 & 0.53 & 0.42 & - & 0.28 \\
\hline & 215 & - & 0.08 & 0.50 & 0.22 \\
\hline & 227 & 0.41 & 0.50 & - & 0.33 \\
\hline & 231 & - & - & 0.44 & 0.11 \\
\hline & 235 & - & - & $0.06^{*}$ & - \\
\hline \multicolumn{6}{|c|}{ СpP2202 } \\
\hline & 188 & 0.44 & 0.46 & 0.50 & 0.55 \\
\hline & 196 & 0.09 & 0.04 & - & - \\
\hline \multirow{2}{*}{\multicolumn{6}{|c|}{ CpDi06 }} \\
\hline & & & & & \\
\hline & 224 & - & - & 0.53 & 0.11 \\
\hline & 226 & $0.02 *$ & - & - & - \\
\hline & 228 & $0.03 *$ & - & - & - \\
\hline & 230 & 0.56 & 0.54 & - & 0.06 \\
\hline & 242 & - & - & $0.47 *$ & - \\
\hline & 244 & - & - & - & $0.11^{*}$ \\
\hline & 246 & 0.05 & - & - & 0.22 \\
\hline & 248 & 0.34 & 0.46 & - & 0.11 \\
\hline & 258 & - & - & - & $0.06 *$ \\
\hline & 260 & - & - & - & $0.06^{*}$ \\
\hline & 262 & - & - & - & $0.06^{*}$ \\
\hline & 264 & - & - & - & $0.22 *$ \\
\hline \multicolumn{6}{|c|}{ CpP208 } \\
\hline & 179 & 0.69 & 0.54 & 0.53 & 0.56 \\
\hline & 191 & 0.31 & 0.46 & 0.47 & 0.44 \\
\hline \multicolumn{6}{|c|}{ СpP314 } \\
\hline & 238 & $0.02 *$ & - & - & - \\
\hline & 242 & $0.02 *$ & - & - & - \\
\hline & 246 & - & - & $0.09 *$ & - \\
\hline & 254 & 0.03 & - & 0.16 & - \\
\hline & 258 & 0.03 & - & 0.63 & - \\
\hline & 262 & - & - & 0.06 & 0.10 \\
\hline & 266 & 0.11 & 0.04 & 0.03 & 0.10 \\
\hline & 270 & 0.22 & 0.31 & - & 0.50 \\
\hline & 274 & 0.06 & 0.27 & 0.03 & 0.10 \\
\hline & 278 & $0.03 *$ & - & - & - \\
\hline & 282 & 0.47 & 0.38 & - & 0.20 \\
\hline & 286 & $0.02 *$ & - & - & - \\
\hline \multicolumn{6}{|c|}{ СpP4311 } \\
\hline & 175 & $0.03 *$ & - & - & - \\
\hline & 187 & 0.22 & 0.08 & - & - \\
\hline & 195 & - & - & 0.78 & 0.22 \\
\hline & 199 & 0.53 & 0.81 & 0.06 & - \\
\hline & 203 & $0.03 *$ & - & - & - \\
\hline & 211 & 0.02 & 0.04 & 0.13 & 0.78 \\
\hline & 215 & $0.08^{*}$ & - & - & - \\
\hline & 219 & 0.09 & 0.08 & 0.03 & - \\
\hline \multicolumn{6}{|c|}{ CpP815 } \\
\hline & 206 & - & - & $0.06^{*}$ & - \\
\hline & 210 & - & - & $0.06^{*}$ & - \\
\hline & 214 & - & - & $0.06^{*}$ & - \\
\hline & 218 & 0.03 & - & 0.22 & 0.10 \\
\hline & 222 & - & - & 0.41 & 0.10 \\
\hline & 226 & 0.06 & - & 0.13 & 0.15 \\
\hline & 230 & 0.31 & 0.35 & 0.03 & - \\
\hline & 234 & - & - & - & $0.10^{*}$ \\
\hline & 238 & 0.17 & 0.08 & 0.03 & 0.40 \\
\hline & 242 & - & $0.19^{*}$ & - & - \\
\hline & 246 & 0.42 & 0.38 & - & 0.15 \\
\hline
\end{tabular}




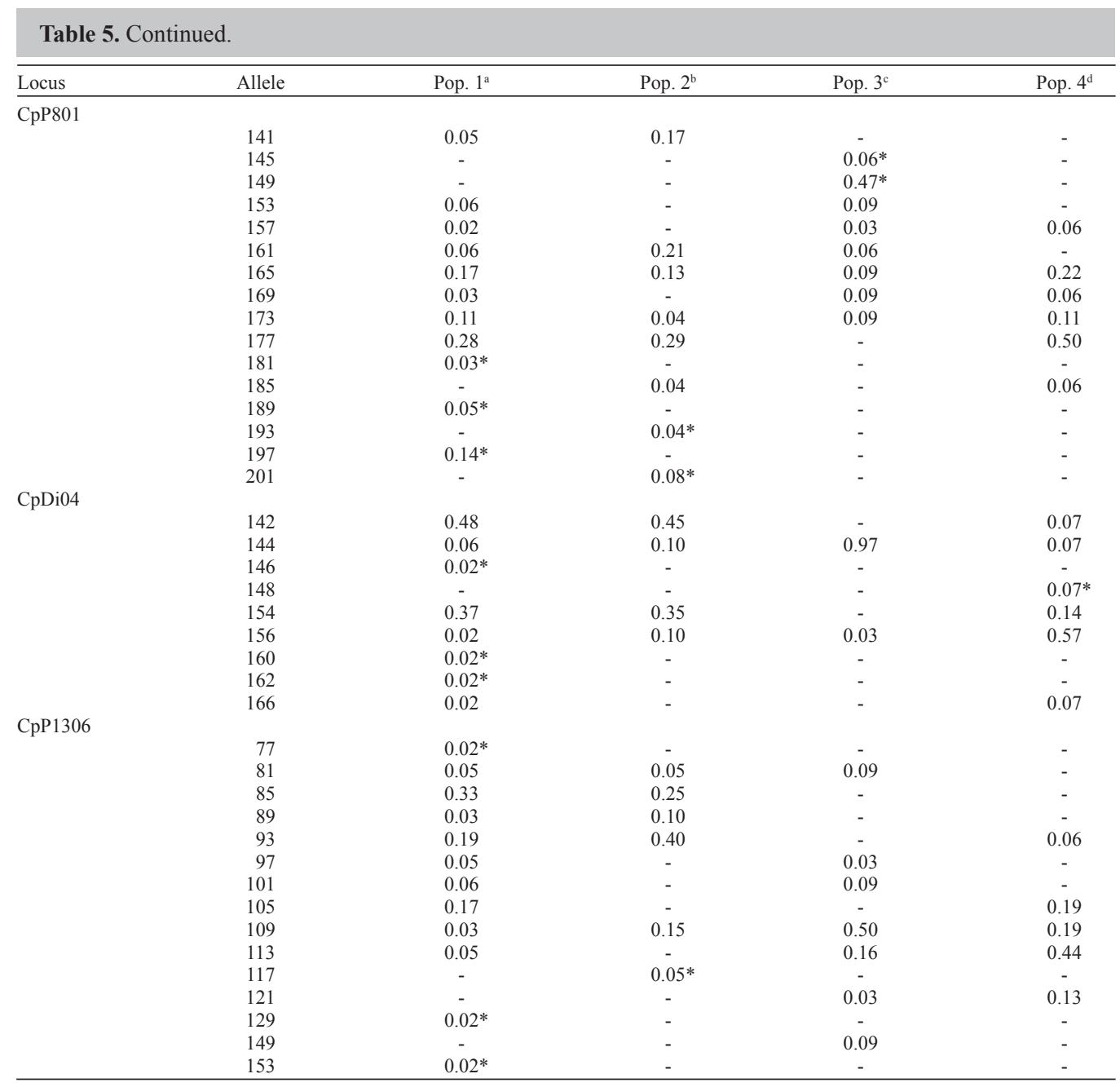

${ }^{\mathrm{a} C}$. moreletii - Belize $(\mathrm{N}=32) ;{ }^{\mathrm{b}}$ C. moreletii - Mexico $(\mathrm{N}=13) ;{ }^{\mathrm{c}}$ C. acutus - Panama $(\mathrm{N}=16) ;{ }^{\mathrm{d}}$ C. acutus - Mexico $(\mathrm{N}=10) . *$ Private allele for that particular locus in that particular population.

\section{ACKNOWLEDGMENTS}

We thank N. Herrera, D. Doens, F. Cano, A.E. Garzón, L. Perez, M.L. Prechi, and E. Lopez at the Smithsonian Tropical Research Institute in Panama for assistance in the laboratory and molecular analysis. We thank J. Edwards, J. Isom, and L. Durham for administrative assistance. We thank 2 anonymous reviewers for their helpful advice and comments to a previous version of this manuscript, and we also thank Dr. S. Schlesinger from the Department of Molecular Microbiology at the Washington University School of Medicine in St. Louis, Missouri, and S. Roussos, E. Mack, M. Sager, and S.A. Baiagurea-Relna from the Densmore Lab for editorial advice and the Sustainable Sciences Institute and Smithsonian Tropical Research 
Institute for their support. Research supported in part by a Howard Hughes Medical Institute grant through the Undergraduate Science Education Program to Texas Tech University (to L.D. Densmore III) and the East Texas Herpetological Society (to B.A. Gross).

\section{REFERENCES}

Anmarkrud JA, Kleven O, Bachmann L and Lifjeld JT (2008). Microsatellite evolution: Mutations, sequence variation, and homoplasy in the hypervariable avian microsatellite locus HrU10. BMC Evol. Biol. 8: 138.

Avise JC (2004). Molecular Markers, Natural History, and Evolution. Sinauer Associates, Sunderland.

Castric V and Bernatchez L (2003). The rise and fall of isolation by distance in the anadromous brook charr (Salvelinus fontinalis Mitchill). Genetics 163: 983-996.

Cedeño-Vázquez JR, Platt SG, Thorbjarnarson J and IUCN Crocodile Specialist Group (2012). Crocodylus Moreletii. In: IUCN 2012. IUCN Red List of Threatened Species. Version 2012.2. Available at [http://iucnredlist.org]. Accessed December 29, 2012.

Crocodile Specialist Group (1996). Crocodylus intermedius. In: IUCN 2012. IUCN Red List of Threatened Species. Version 2012.2. Available at [http://iucnredlist.org]. Accessed December 29, 2012.

Davis LM, Glenn TC, Elsey RM, Dessauer HC, et al. (2001). Multiple paternity and mating patterns in the American alligator, Alligator mississippiensis. Mol. Ecol. 10: 1011-1024.

Davis LM, Glenn TC, Strickland DC, Guillette LJ Jr, et al. (2002). Microsatellite DNA analyses support an east-west phylogeographic split of American alligator populations. J Exp. Zool. 294: 352-372.

Dever JA and Densmore III LD (2001). Microsatellite's in Morelet's crocodiles (Crocodylus moreletii) and their utility in addressing crocodilian population genetics questions. J. Herpetol. 35: 541-544.

Dever JA, Strauss RE, Rainwater TR, McMurry ST, et al. (2002). Genetic diversity, population subdivision, and gene flow in Morelet's crocodile (Crocodylus moreletii) from Belize, Central America. Copeia 4: 1078-1091.

Fitzsimmons N, Tanksley S, Forstner MRJ, Louis EE, et al. (2001). Microsatellite Markers for Crocodylus: New Genetic Tools for Population Genetics, Mating System Studies and Forensics. In: Crocodilian Biology and Evolution (Grigg G, Seebacher F and Franklin C, eds.). Surrey Beatty and Sons, Chipping Norton, 51-56.

Glenn TC, Dessauer HC and Braun MJ (1998). Characterization of microsatellite DNA loci in American alligators. Copeia 3: 591-601.

Gunn MR, Dawson DA, Leviston A, Hartnup K, et al. (2005). Isolation of 18 polymorphic microsatellite loci from the North American red squirrel, Tamiasciurus hudsonicus (Sciuridae, Rodentia), and their cross-utility in other species. Mol. Ecol. Notes 5: 650-653.

Kalinowski ST (2004). Counting alleles with rarefaction: private alleles and hierarchical sampling designs. Conserv. Genet. 5: 539-543.

King RB (2009). Population and Conservation Genetics. In: Snakes: Ecology and Conservation (Mullin SJ and Seigel RA, eds.). Cornell University Press, New York, 78-122.

King TL, Lubinski BA and Spidle AP (2001). Microsatellite DNA variation in Atlantic sturgeon (Acipenser oxyrinchus oxyrinchus) and cross-species amplification in the Acipenseridae. Conserv. Genet. 2: 103-119.

Marshall TC, Slate J, Kruuk LE and Pemberton JM (1998). Statistical confidence for likelihood-based paternity inference in natural populations. Mol. Ecol. 7: 639-655.

Miles LG, Isberg SR, Moran C, Hagen C, et al. (2009a). Two hundred and fifty-three novel polymorphic microsatellites for the saltwater crocodile (Crocodylus porosus). Conserv. Genet. 10: 963-980.

Miles LG, Lance SL, Isberg SR, Moran C, et al. (2009b). Cross-species amplification of microsatellites and applications for the future. Conserv. Genet. 10: 935-954.

Milián-Garcia Y, Venegas-Anaya M, Frias-Soler R, Crawford AJ, et al. (2011). Evolutionary history of Cuban crocodiles Crocodylus rhombifer and Crocodylus acutus inferred from multilocus markers. J. Exp. Zool. A Ecol. Genet. Physiol. 315: 358-375.

Palumbi SR (1996). Nucleic Acids II: The Polymerase Chain Reaction. In: Molecular Systematics. 2nd edn. (Hillis DM, Moritz C and Mable BK, eds.). Sinauer Associates, Sunderland.

Peakall R and Smouse PE (2006). GENALEX 6: genetic analysis in Excel. Population genetic software for teaching and research. Mol. Ecol. Notes 6: 288-295.

Ponce-Campos P, Thorbjarnarson J, Velasco A and IUCN SSC Crocodile Specialist Group (2012). Crocodylus Acutus. In: IUCN 2012. IUCN Red List of Threatened Species. Version 2012.2. Available at [http://iucnredlist.org]. Accessed December 29, 2012. 
Primmer CR, Moller AP and Ellegren H (1996). A wide-range survey of cross-species microsatellite amplification in birds. Mol. Ecol. 5: 365-378.

Primmer CR, Painter JN, Koskinen MT, Palo JU, et al. (2005). Factors affecting avian cross-species microsatellite amplification. J. Avian Biol. 36: 348-360.

Rodriguez D, Cedeno-Vazquez JR, Forstner MR and Densmore LD III (2008). Hybridization between Crocodylus acutus and Crocodylus moreletii in the Yucatan Peninsula: II. Evidence from microsatellites. J. Exp. Zool. A Ecol. Genet. Physiol. 309: 674-686.

Rodriguez D, Forstner MRJ, Moler PE, Wasilewski JA, et al. (2011). Effect of human-mediated migration and hybridization on the recovery of the American crocodile in Florida (USA). Conserv. Genet. 12: 449-459.

Sambrook J, Fritsch EF and Maniatis T (1989). Molecular Cloning: A Laboratory Manual, 2nd edn. Cold Spring Harbor Laboratory Press, New York, 1.21-1.29.

Schuelke M (2000). An economic method for the fluorescent labeling of PCR fragments. Nat. Biotechnol. 18: 233-234.

Scribner KM, Gust JR and Fields RL (1996). Isolation and characterization of novel salmon microsatellite loci: crossspecies amplification and population genetic applications. Can. J. Fish. Aquat. Sci. 53: 833-841.

Skotheim RI, Diep CB, Kraggerud SM, Jakobsen KS, et al. (2001). Evaluation of loss of heterozygosity/allelic imbalance scoring in tumor DNA. Cancer Genet. Cytogenet. 127: 64-70.

Targarona RR, Soberón RR, Cotayo L, Tabet MA, et al (1996). Crocodylus rhombifer. In: IUCN 2012. IUCN Red List of Threatened Species. Version 2012.2. Available at [http://iucnredlist.org]. Accessed December 29, 2012.

Vandewege MW, Rodriguez D, Weaver JP, Hibbitts TD, et al. (2012). Evidence of hybridization between Elaphe bairdi and Elaphe obsolete lindheimeri including comparative population genetics inferred from microsatellites and mitochondrial DNA. J. Herpetol. 46: 56-63.

Watzinger F, Lion T and Steward C (2006). The RSD code: proposal for a nomenclature of allelic configurations in STRPCR-based chimerism testing after allogeneic stem cell transplantation. Leukemia 20: 1448-1452.

Weaver JP, Rodriguez D, Venegas-Anaya M, Cedeno-Vazquez JR, et al. (2008). Genetic characterization of captive Cuban crocodiles (Crocodylus rhombifer) and evidence of hybridization with the American crocodile (Crocodylus acutus). J. Exp. Zool. A Ecol. Genet. Physiol. 309: 649-660.

Williamson JE, Huebinger RM, Sommer JA, Louis EE, et al. (2002). Development and cross-species amplification of 18 microsatellite markers in the Sumatran tiger (Panthera tigris sumatrae). Mol. Ecol. Notes 2: 110-112.

Wilson ACC, Massonnet B, Simon JC, Prunier-Leterme N, et al. (2004). Cross-species amplification of microsatellite loci in aphids: assessment and application. Mol. Ecol. Notes 4: 104-109.

Wright JM and Bentzen P (1994). Microsatellites: genetic markers for the future. Rev. Fish. Biol. Fisher. 4: 384-388.

Zucoloto RB, Villela PMS, Verdade LM and Coutinho LL (2006). Cross-species microsatellite amplification in South American Caimans (Caiman spp and Paleosuchus palpebrosus). Genet. Mol. Biol. 29: 75-78. 